Gerhard Illing, Sebastian Jauch und Michael Zabel ${ }^{1}$

\title{
Die Diskussion um den Euro
}

Endogene Risiken und multiple Gleichgewichte

»Vereinfacht lässt sich sagen: Jeder Porsche, der nach Griechenland geliefert wird, wird derzeit von Deutschland bezahlt. « (Blankart 2012)

Die deutsche Debatte über die Eurokrise unterscheidet sich stark von der internationalen Diskussion. ${ }^{2}$ Weil bei dem Deutungsmuster, das von namhaften deutschen Ökonomen, Politikern und Notenbankern vertreten wird, die wahren Ursachen der Krise verkannt werden, laufen zahlreiche deutsche Vorstöße Gefahr, kontraproduktiv zu wirken und die Bemühungen um eine Stabilisierung in den kriselnden Euroländern zu konterkarieren. Beispielhaft dafür ist der Verlauf der aktuellen Debatte um die Risiken des Clearingsystems Target $2^{3}$. Es werden Probleme benannt, ohne deren Ursachen gründlich zu beleuchten, bestimmte Maßnahmen isoliert kritisiert, ohne deren Gesamtzusammenhang zu berücksichtigen, und Lösungsansätze präsentiert, ohne deren Konsequenzen klar zu benennen. Ziel dieses Beitrags ist es, auf Grundlage einer breiten Analyse der Ursachen der Krise die Risiken der derzeitigen Diskussion aufzuzeigen und die Kurzsichtigkeit mancher Argumentationsstrukturen deutlich zu machen.

\section{Keine Staatsverschuldungskrise}

Lange Zeit wurde, geprägt von der Entwicklung in Griechenland, das Hauptproblem im Euroraum in den hohen Staatsschulden gesehen. Verglichen mit anderen Industrienationen wie den USA, Großbritannien oder Japan liegt die Staatsverschuldung des Euroraums jedoch auf vergleichsweise niedrigem Niveau (vgl. Abbildung 1). Zudem ist die Schuldenquote in den meisten betroffenen Ländern seit der globalen Finanzkrise vor allem deshalb gestiegen, weil einerseits die Wirtschaftsleistung zurückging und andererseits Bankenrettungs- und Konjunkturprogramme finanziert werden mussten. Daher handelt es sich bei der aktuellen Staatsschuldenproblematik (vom Ausnahmefall Griechenland abgesehen) nicht um ein sich seit langem abzeichnendes Problem, das durch freigiebige Staatsausgabenpoli-

1 Die Autoren danken zwei anonymen Gutachtern für konstruktive Kommentare.

2 Vgl. etwa De Grauwe (2011); Eichengreen (2012); Sargent (2011).

3 Das Clearingsystem Target2 ist das Zahlungssystem zwischen den nationalen Notenbanken und dient der Abwicklung von Überweisungen in Echtzeit. 
Abbildung 1: Entwicklung der Staatsschuldenquote im Euroraum und in unterschiedlichen Industriestaaten

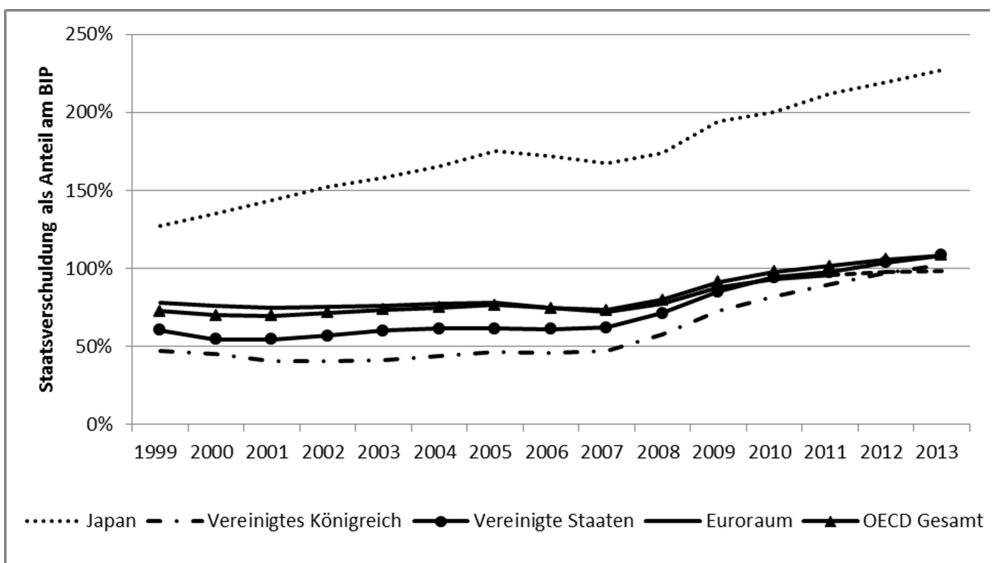

Quelle: OECD Economic Outlook November 2011.

tik verursacht wurde. Krisenländer wie Irland und Spanien haben (im Gegensatz zu Deutschland) in den Vorkrisenjahren die Kriterien des Stabilitäts- und Wachstumspakts vielmehr mustergültig eingehalten (vgl. Abbildung 2), also die »technischen Anforderungen « an die Mitglieder der Eurozone erfüllt. Ja, deren Regierungen gelang es sogar über mehrere Jahre hinweg, Budgetüberschüsse auszuweisen - was in Deutschland seit Beginn der 1970er Jahre nur ein einziges Mal vorgekommen ist.

\section{Abbildung 2: Entwicklung der Staatsschuldenquote in ausgewählten Ländern des Euroraums}

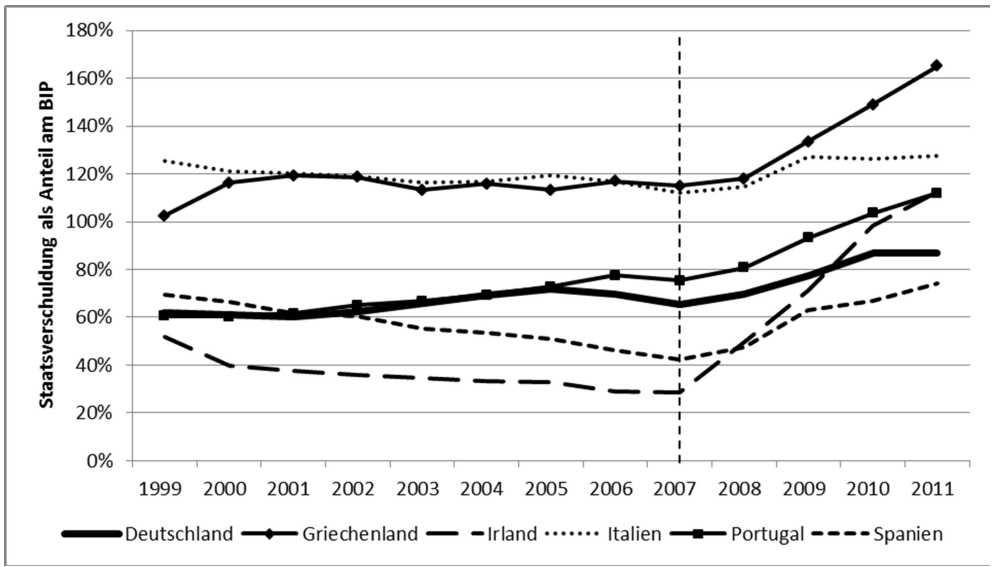

Quelle: OECD Economic Outlook November 2011.

Leviathan, 40. Jg., 2/2012 
Das Niveau der Staatsschulden liefert damit keine hinreichende Erklärung für die aktuellen Probleme des Euroraums. Ausschlaggebend ist vielmehr eine dramatische Umkehr der Kapitalströme im Euroraum seit Ausbruch der Finanzkrise, kombiniert mit mangelnder Wettbewerbsfähigkeit der Länder in der Peripherie. Die Liberalisierung der Finanzmärkte hatte in den Anfangsjahren des Euro einen rasanten $\mathrm{Zu}$ strom privaten Kapitals in die Peripherieländer ausgelöst. Kapital floss nach Spanien, Irland und Italien in der Erwartung wirtschaftlicher Konvergenz aufgrund einheitlicher und stabiler Rahmenbedingungen innerhalb des Euroraums. Die Anleger vertrauten darauf, dass die politischen Institutionen im ganzen Euroraum höchsten Qualitätsstandards genügen. Die Finanzkrise hat dieses Vertrauen stark erschüttert. Jede plötzliche Umkehr der Kapitalströme löst in Defizitländern zwangsläufig einen massiven Anpassungsdruck aus, während Überschussregionen umgekehrt zunächst einmal als sichere Häfen davon profitieren. Die Möglichkeit, Finanzkapital elektronisch per Knopfdruck jederzeit schnell und ohne Wechselkursrisiken im gesamten Euroraum umschichten zu können, birgt aber die Gefahr der Destabilisierung des gesamten Systems. Während es in den Anfangsjahren der Währungsunion zu einem Überschießen der Kapitalströme in Richtung Peripherie gekommen ist, droht nun, im umgekehrten Prozess, eine explosive Dynamik in Gang zu kommen: Moderne Finanzintermediation hat es im Übermaß erleichtert, langfristige, illiquide Anlagen durch kurzfristige, jederzeit abziehbare Einlagen zu finanzieren. ${ }^{4}$ In einer Krise erscheint es für jeden einzelnen Anleger individuell rational, seine Einlagen abzuziehen. Der massive Abzug solcher Einlagen führt aber zum Zusammenbruch der gesamten Wirtschaftsaktivität und gefährdet damit die Rückzahlung aus den entsprechenden Investitionsprojekten.

Manche interpretieren den Abzug des Kapitals aus den Defizitstaaten als Antwort rationaler Anleger auf die Erkenntnis fehlender Wettbewerbsfähigkeit dieser Regionen. Als Maß für die Wettbewerbsfähigkeit gilt oftmals die Entwicklung der Lohnstückkosten. In der Tat haben sich diese seit der Einführung des Euro sehr unterschiedlich entwickelt. So blieben sie in Deutschland über zehn Jahre nahezu konstant, während sie in den heutigen Problemländern stark angestiegen sind (vgl. Abbildung 3). Bei dieser Betrachtungsweise wird jedoch leicht übersehen, dass zum Zeitpunkt der Euroeinführung die absoluten Niveaus der Lohnstückkosten stark divergierten. Die Entwicklung der Lohnstückkosten ist also nicht ausschließlich einer maßlosen Lohnpolitik in den Peripheriestaaten geschuldet. $\mathrm{Zu}$ einem großen Teil lässt sie sich vielmehr auf die Reduktion von Divergenzen im Euroraum zurückführen. Entsprechend dem Balassa-Samuelson-Effekt profitieren Regionen mit niedrigerer Kapitalausstattung nach einer Liberalisierung der Finanzmärkte von steigender Produktivität dank des Kapitalzustroms: Die stärker steigende Arbeitsproduktivität führt als Folge freier Marktbewegungen zu einer Angleichung der Lohnstückkosten - ein ausdrückliches politisches Ziel der europäischen Integration.

4 Ein Beispiel für diese Entwicklung ist der rasante Anstieg von Kreditverbriefungen. Kreditverbriefungen ermöglichen es, Kredite zu bündeln, in Tranchen mit unterschiedlichem Risikotyp aufzuteilen und so ein scheinbar liquides Konstrukt am Kapitalmarkt zu handeln. 
Abbildung 4: Entwicklung der Arbeitslosenquoten in ausgewählten Ländern des Euroraums

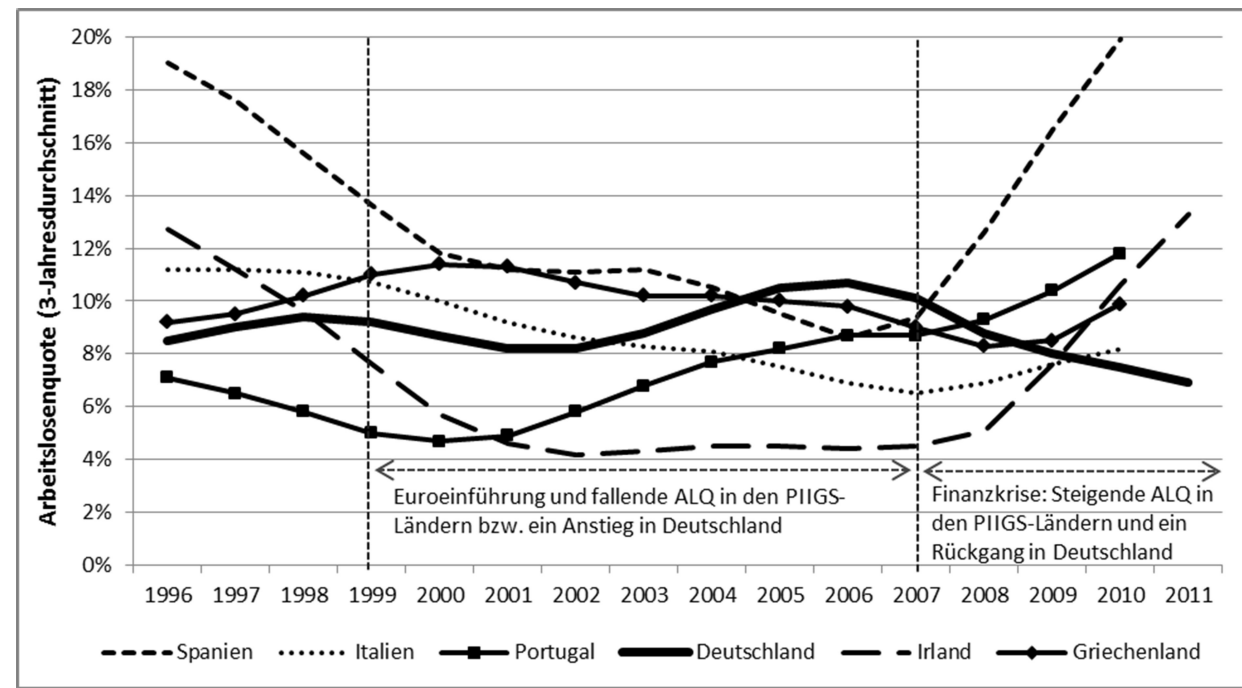

Quelle: Eurostat.

\section{Abbildung 3: Relative Entwicklung der Lohnstückkosten im Euroraum}

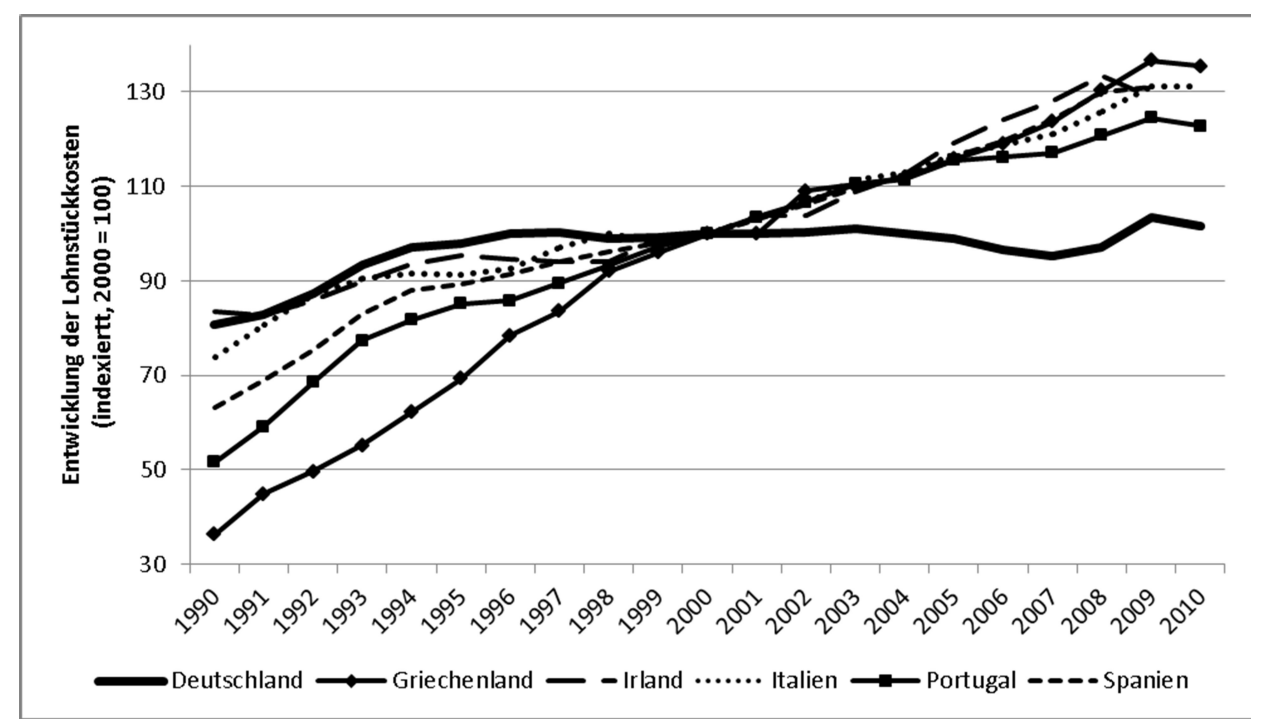

Quelle: OECD Economic Outlook November 2011. 
Der Verlauf der Arbeitslosenquoten seit der Euroeinführung korrespondiert eindrucksvoll mit der Einschätzung der Wettbewerbsfähigkeit im Euroraum. Zu Beginn des Jahrtausends war auf der einen Seite die deutsche Wirtschaft durch eine hohe, persistente Arbeitslosigkeit gekennzeichnet (interpretiert als Folge geringer Wettbewerbsfähigkeit; Deutschland wurde damals auch als »kranker Mann Europas « bezeichnet), während umgekehrt die Arbeitslosenquoten in den meisten Ländern der Peripherie stetig zurückgingen (vgl. Abbildung 4). Forciert wurde dieser Prozess durch private Kapitalströme aus den Kernländern, insbesondere aus Deutschland. Vor der Krise wurde diese Entwicklung vielfach als Indiz einer effizienten Marktallokation gepriesen. Der stetige Kapitalfluss in die PIIGS-Staaten ${ }^{5}$ (zwangsläufig einhergehend mit hohen Leistungsbilanzdefiziten) wurde als Zeichen der Wachstumskraft dieser Regionen interpretiert, die Kapitalabflüsse aus Deutschland spiegelbildlich als Konsequenz der dortigen Wachstumsschwäche.

Seit Ausbruch der Finanzkrise hat sich das Bild schlagartig verändert: Die stark angestiegenen Arbeitslosenquoten in den Problemstaaten werden nun ebenso wie die dort auch nach mehreren Jahren Krise weiterhin bestehenden Leistungsbilanzdefizite als untrügliches Indiz ihrer chronischen mangelnden Wettbewerbsfähigkeit gesehen. Wie konnte es zu einer solch dramatischen Umkehr kommen? Die Leistungsbilanzungleichgewichte haben sich schon seit der Euroeinführung aufgebaut; sie hätten somit schon von Anfang an zu Skepsis herausfordern müssen. Offensichtlich ist es sowohl bei den Kapitalströmen wie bei der Lohnentwicklung im Euroraum im Lauf des letzten Jahrzehnts zu einem Überschießen gekommen. Wer heute die anfänglichen Kapitalflüsse in die Peripheriestaaten und die dortige Entwicklung am Arbeitsmarkt als Fehlentwicklung ansieht, sollte vorsichtig dabei sein, die drastische Umkehr der Kapitalströme nunmehr als Zeichen funktionierender Marktkräfte zu interpretieren. Das freie Spiel der Marktkräfte entfaltet nur bei angemessenen staatlichen Rahmenbedingungen die gewünschte Wirkung; ohne stabile Ordnung und vernünftige Regulierung der Finanzmärkte können die Marktkräfte Fehlentwicklungen mit fataler Wucht verstärken.

\section{Wettbewerbsfähigkeit, Kapitalströme und Target2-Salden}

Studenten der Wirtschaftswissenschaften lernen im ersten Studienjahr, dass Kapital dorthin fließt, wo die höchste Grenzproduktivität erzielt werden kann. Wenn die Renditen auf Kapital in Spanien höher sind als in Deutschland, sind Nettokapitalflüsse von Deutschland nach Spanien das Resultat einer effizienten Allokation limitierter Ressourcen. In dieser Sichtweise wird jedoch der Anteil der Rendite ignoriert, der auf eine Blasenbildung zurückzuführen ist.

Aus der Perspektive eines rationalen Individuums kann es durchaus schlüssig sein, einem Herdentrieb zu folgen und getreu dem Motto »the trend is my friend « tem-

5 Die PIIGS-Staaten stehen für die Länder in der Peripherie des Euroraums, die von der Krise besonders betroffen sind - Portugal, Italien, Irland, Griechenland und Spanien. Manchmal wird auch, ohne Italien einzubeziehen, von den PIGS- bzw. GIPS-Staaten gesprochen. 
porär in eine entstehende Vermögenswertblase zu investieren. Aus aggregierter Sicht stellt dies jedoch eine ungesunde Entwicklung dar, wenn der Wertentwicklung keine entsprechenden Fundamentaldaten gegenüberstehen, wenn also Anlagewerte inflationiert sind. Das Platzen der Immobilienblasen in Spanien und Irland hat auf schmerzhafte Weise klargemacht, dass die Kapitalströme in diese Länder nicht nur zum Aufbau benötigter produktiver Infrastruktur, für Investitionen in Bildung und zur Finanzierung produktiver Anlagen verwendet wurden. Das Kapital deutscher Sparer hat auch unproduktive und damit unrentable Projekte ermöglicht. Dieser Kapitalzustrom in die Peripherie beruhte auf der Illusion einer hohen Wettbewerbsfähigkeit und der Hoffnung, dass sich diese zukünftig in guten Renditen widerspiegelt. Gestützt wurde diese Entwicklung durch das Vertrauen in die europäischen Institutionen und deren Unterstützung des wirtschaftlichen Konvergenzprozesses im Euroraum. Aufgrund dieser Einschätzung in den Überschussländern war es Bürgern und Unternehmen in den Ländern mit Leistungsbilanzdefiziten möglich, sich über die rentablen Projekte hinaus zu verschulden und in der Hoffnung auf ein zukünftiges hohes Einkommen Ausgaben über Kredit zu finanzieren. Die Kapitalmärkte allozieren Kapital auf der Grundlage von Erwartungen über zukünftige Entwicklungen. Das Beispiel deutscher Kapitalströme in die Peripheriestaaten macht aber deutlich, dass Anleger bei ihrer Erwartungsbildung zu Übertreibungen neigen, was zu Wohlfahrtsverlusten führt. Offensichtlich verleitet zudem die Hoffnung, Mittel rechtzeitig wieder abziehen zu können, zu Kurzfristdenken auf den Kapitalmärkten und löst damit Marktversagen aus.

Das Ende der überschätzten Wettbewerbsfähigkeit kam mit den Schockwellen der amerikanischen Subprimekrise. Investoren aus den Überschussländern erkannten die Übertreibungen im amerikanischen Immobilienmarkt und begannen unter diesem Eindruck, Kapital aus den Peripherieländern des Euroraums abzuziehen. Die plötzliche Umkehrung der Kapitalströme entspricht einem »sudden stop", der auch Auslöser von Krisen in Schwellenländern (Emerging Markets) nach einer Liberalisierung der Finanzmärkte war, wie man es in Lateinamerika in den 1970er Jahren und in der Asienkrise Ende der 1990er Jahre beobachten konnte. Wenn Investoren ihr Kapital gleichermaßen aus unproduktiven und produktiven Anlagen abziehen, werden einerseits Übertreibungen sichtbar und Blasen platzen, andererseits werden durch die Abflüsse auch produktives Kapital und intakte Wirtschaftsstrukturen zerstört, die aufgrund des allgemeinen Misstrauens keine tragfähige Kreditfinanzierung mehr bekommen, auf die sie aber angewiesen sind. Einer Übertreibung nach oben, die in die wahllose Finanzierung ungenügend geprüfter Projekte mündet, folgt quasi eine Übertreibung nach unten mit dem massiven Abzug von Kapital unabhängig von dessen Verwendungsart.

Die Kapitalabflüsse führen zu einer Kreditklemme, welche neben langfristigen Investitionen auch kurzfristige Lieferantenkredite erschwert. Groß- und Kleinunternehmen fällt es schwerer, in Vorleistung zu treten und ihre Geschäfte zu finanzieren. Bei manchen Betrieben wird dies eine ohnehin wahrscheinliche Insolvenz früher eintreten lassen, bei anderen ist die so erzeugte Illiquidität jedoch erst der Auslöser einer Insolvenz. Insolvenzen infolge einer Kreditklemme führen dazu, dass sich das gesamtwirtschaftliche Gleichgewicht auf einem niedrigeren Einkommens- und Pro- 
duktionsniveau einstellt. Der dramatische Einbruch des Wachstums der Geldmenge M2 in den Krisenstaaten ist ein klarer Indikator für die Existenz einer Kreditklemme (vgl. Abbildung 5). Die heutige Entwicklung im Euroraum ist eine beängstigende Bestätigung des Phänomens multipler Gleichgewichte: Bei Vertrauen, intakten Wirtschaftsstrukturen mit geordneter Kreditfinanzierung und geringer Arbeitslosigkeit realisiert sich das gute Gleichgewicht. Dagegen stellt sich ohne Vertrauen in stabile Institutionen und bei einer Umkehr der Kreditfinanzierung ein schlechtes Gleichgewicht ein mit maroden Wirtschaftsstrukturen und hoher Arbeitslosigkeit.

Abbildung 5: Entwicklung des Wachstums der Geldmenge M2 in den europäischen Krisenstaaten

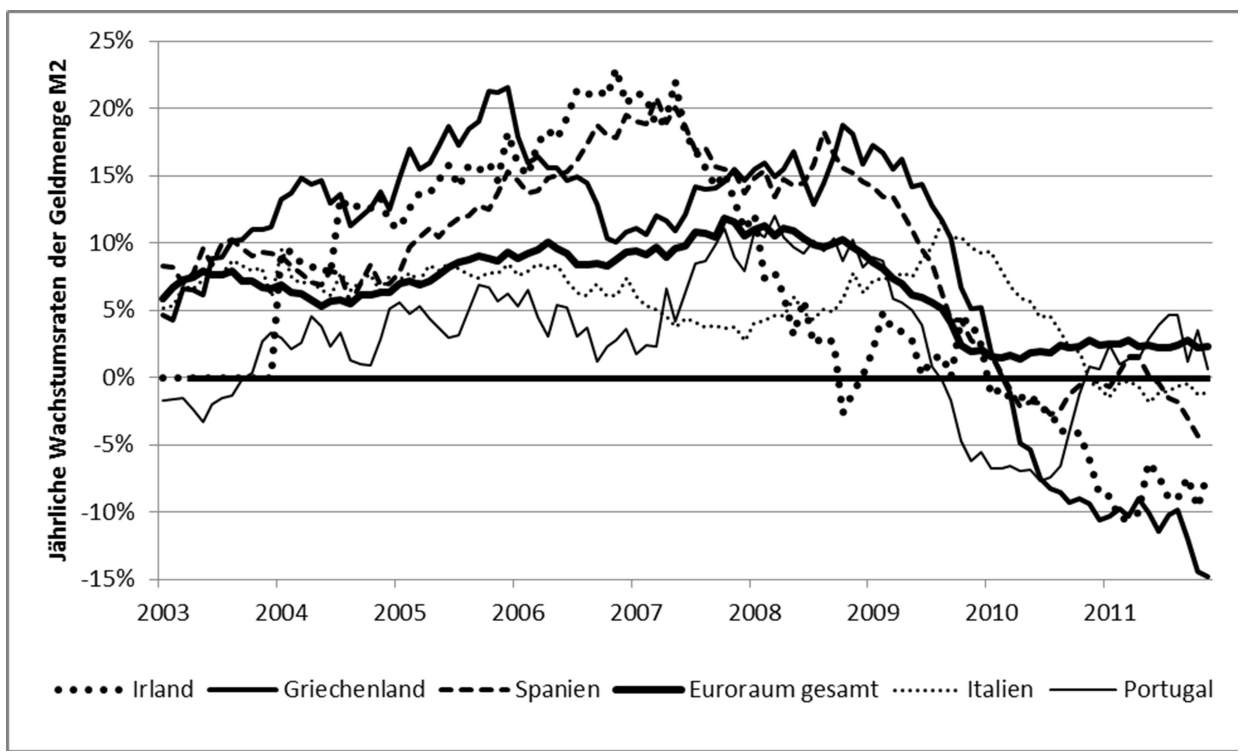

Quelle: Nationale Zentralbanken des Eurosystems.

Der Einbruch der Wirtschaftsaktivität in den Peripherieländern ist nicht zuletzt Konsequenz eines Vertrauensverlustes, der diese Länder in das schlechtere der möglichen Gleichgewichte führt. Dieser schlechte Zustand wird zementiert, da das fehlende Vertrauen und die Ungewissheit über die politische und monetäre Zukunft Investitionen unattraktiv machen, das Konsumverhalten der Bürger bremst und so eine Negativspirale bei der Wirtschaftsentwicklung in Gang kommt. Diese Abwärtsspirale zu durchbrechen ist die zentrale Herausforderung der Politiker in Brüssel, Berlin und Athen.

Um einen vollständigen »sudden stop « und damit eine noch schlimmere Krise zu verhindern, müssen überstürzte private Kapitalabflüsse gebremst oder durch andere Zuflüsse kompensiert werden. Privates Kapital kann nur über Vertrauen und positive Zukunftsaussichten im Land gehalten werden. Diese Voraussetzungen haben 
bislang weder Athen noch Brüssel oder die Europäische Zentralbank (EZB) in Frankfurt geschaffen. Die einzige Möglichkeit, dramatische Auswirkungen der privaten Kapitalabflüsse zu verhindern, besteht im Ersatz durch staatliche Kapitalzuflüsse. Genau dies geschieht derzeit im Euroraum.

Mit dem Aufbau von Salden zwischen den Einheiten des Eurosystems wird ein Kapitalabfluss aus den Ländern der Peripherie verhindert. Um es an einem Beispiel zu verdeutlichen: Löst ein italienisches Unternehmen sein Konto in Italien auf und transferiert das Geld auf ein Konto in Deutschland, haben sowohl die deutsche als auch die italienische Bank weiterhin unverändert ausstehende Kreditvolumina. Wenn die deutsche Bank, der das Geld des italienischen Unternehmers zugeflossen ist, ihre überschüssigen Einlagen nun über die Bundesbank bei der EZB parkt, kann die EZB diese Einlagen über die italienische Zentralbank wieder der italienischen Bank zur Verfügung stellen, die den Abfluss zu verzeichnen hatte. Der Aufbau von Salden innerhalb des Eurosystems verhindert so den Ansturm auf Banken, die auch im gesündesten Zustand nur begrenzt Liquiditätsabflüsse verkraften können. Diesem Ausgleichssystem ist es zu verdanken, dass italienische Banken trotz Abflüssen von über 200 Milliarden Euro innerhalb weniger Monate keinem »Bank Run « ausgesetzt waren. Bei den Ausgleichszahlungen innerhalb des Eurosystems (den sogenannten Target2-Salden) handelt es sich somit keineswegs um ineffiziente Staatseingriffe, die ein reinigendes Gewitter verhindern, sondern um Maßnahmen, die eine Kapitalknappheit bei den privaten Banken abschwächen, welche über andernfalls drohende Illiquidität zu Insolvenzen führen würde.

In der deutschen Öffentlichkeit wird häufig der Eindruck erweckt, mit diesen Ausgleichszahlungen würde ein übermäßiger Konsum der Bürger in der Peripherie finanziert. Der deutsche Steuerzahler, der letztlich für Verbindlichkeiten der deutschen Bundesbank haftet, finanziere so das schöne Leben am Mittelmeer. Bei den Target2-Salden handelt es sich jedoch um einen Ausgleich der Kapitalabflüsse. Würden die Kredite dieser Target2-Salden zur Finanzierung von Nettoimporten in den Krisenstaaten verwendet, müsste dies an einer starken Korrelation mit der Leistungsbilanz sichtbar werden. Wenn also eine Zentralbank im Mittelmeerraum sich über die EZB bei der Bundesbank Geld leiht, es an die eigenen Bürger weitergibt und diese dafür mehr Güter kaufen, als sie selbst produzieren, müsste der Überschuss der Importe über die Exporte genau die Veränderung des Target2-Saldos der entsprechenden Periode wiedergeben. Eine ökonometrische Überprüfung der Korrelation zwischen der Leistungsbilanz und der Veränderung des Target2-Saldos eines Landes lässt jedoch einen solchen Zusammenhang nicht erkennen. ${ }^{6}$ So weist etwa Irland den höchsten Target2-Saldo auf, gemessen als Anteil am BIP, erzielt aber inzwischen sogar Leistungsbilanzüberschüsse. Tabelle 1 zeigt die Korrelationskoeffizienten für Deutschland und die PIIGS-Länder.

6 Vgl. dazu Bindseil, Koenig 2011; Sinn, Wollmershaeuser 2011.

Leviathan, 40. Jg., 2/2012 
Tabelle 1: Korrelation der Leistungsbilanz mit der Veränderung des Target2-Saldos

\begin{tabular}{lcc}
\hline & Krise $(2008-2011)$ & Signifikant auf $\mathbf{1 0 \% - N i v e a u}$ \\
\hline Deutschland & $-22,8$ & nein \\
Griechenland & 4,9 & nein \\
Irland & $-4,3$ & nein \\
Italien & $-12,3$ & nein \\
Portugal & 14,7 & nein \\
Spanien & $-7,0$ & nein \\
\hline
\end{tabular}

Quelle: Datastream, ifo Institut.

Die Korrelationskoeffizienten zwischen Leistungsbilanzen und den Veränderungen der Target2-Salden sind auf Basis von gleitenden 3-Monatsdurchschnittswerten berechnet. Für Irland beziehen sich die Korrelationskoeffizienten auf Quartalsdaten.

Die Werte in Tabelle 1 zeigen, dass die Korrelationen von Leistungsbilanz und Veränderung des Target2-Saldos sehr gering sind. Vor allem aber sind sie nicht signifikant unterschiedlich von Null. Bei Betrachtung des Zeitraums von der Euroeinführung bis zum Beginn der Krise ergibt sich ein ähnliches Bild. Eine Interpretation der Höhe und des Vorzeichens der Korrelationskoeffizienten wäre aufgrund der fehlenden Signifikanz irreführend.

Sinn und Wollmershaeuser zufolge ${ }^{7}$ ist der Anstieg der Target2-Salden seit Ausbruch der Finanzkrise 2007 darauf zurückzuführen, dass seitdem Zentralbankkredite zu einem beträchtlichen Teil die private Finanzierung von Leistungsbilanzsalden substituieren. Aus dieser Sicht wird jedoch die Brisanz des Vorgangs verkannt, was zu falschen wirtschaftspolitischen Schlussfolgerungen verleitet. Wenn das Kernproblem in der Kapitalflucht liegt, ${ }^{8}$ ergibt sich das Gesamtpotential möglicher Target2-Salden keineswegs aus der seit Ausbruch der Finanzkrise akkumulierten Leistungsbilanz. In einem einheitlichen Währungsraum ausschlaggebend sind dann vielmehr die in der Vergangenheit insgesamt akkumulierten liquiden Finanzmittel. ${ }^{9}$ Der relevante Zusammenhang besteht dann darin, dass der Kapitalausgleich über die Target2-Salden eine überstürzte Auflösung der in der Vergangenheit vergebenen privaten Kredite und so den kompletten Einbruch der Wirtschaftsaktivität und damit auch des Konsums verhindert. Der traditionellen Sicht effizienter Märkte zufolge stellen Leistungsbilanzdefizite kein Problem dar, solange sie sich als Folge privater Kapitalströme ergeben haben. Wie bei allem, was sich freiwillig zwischen Erwachsenen abspielt, sollte es den privaten Marktakteuren überlassen bleiben, wie sie ihre Investitionsentscheidungen fällen. In der realen Welt fragiler Finanzmärkte

7 »Bemerkenswert ist, dass es eine weitgehende, wenn auch nicht perfekte Korrelation zwischen dem Anstieg der deutschen Target-Forderungen und dem Anstieg der Verbindlichkeiten der GIPS-Staaten gab « (Sinn, Wollmershaeuser 2011, S. 4).

8 Zur Bedeutung der Kapitalflucht vgl. auch Bornhorst, Mody 2012.

9 Die Rolle liquider Brutto-Kapitalströme für die systemische Finanzmarktstabilität untersuchen Bruno, Shin 2012. 
ist jedoch ein persistenter Aufbau hoher Leistungsbilanzungleichgewichte nicht unbedingt Resultat guter Fundamentaldaten, sondern kann vielmehr Indiz des Aufbaus starker Krisenanfälligkeit sein. ${ }^{10}$

Ist es also falsch, wenn manche Wirtschaftswissenschaftler Target2-Zahlungen einen Kredit nennen und einen direkten Bezug zwischen den Target2-Salden und dem Konsum der Peripherieländer aufbauen? Nicht unbedingt: Denn obgleich die Ursache der Target2-Salden wie dargestellt nicht im Konsumanstieg, sondern in der Kapitalflucht liegt, gilt natürlich, dass das bestehende Konsumniveau ohne die Target2-Zahlungen nicht aufrechterhalten werden könnte. Der Konsum würde also deutlich niedriger ausfallen. Ein kurzer Analogieschluss veranschaulicht dies: Betrachten wir einen Haushalt, der seine Ersparnisse bei einer Bank angelegt hat. Die Bank gerät nun jedoch in Zahlungsschwierigkeiten. Sie bekommt Liquiditätshilfen von der Zentralbank, so dass sie keine Insolvenz anmelden muss. In diesem Fall verliert der Haushalt nicht seine gesamten Ersparnisse und kann daher weiterhin das gleiche Konsumniveau nachfragen wie zuvor. Da ihm dies aber nur deshalb möglich ist, weil die Zentralbank den Kollaps der Bank verhinderte, wird der Konsum des Haushalts tatsächlich durch einen Kredit der Zentralbank gestützt. Es wäre jedoch verfehlt, zu behaupten, der Haushalt würde nun auf Kosten der Zentralbank mehr konsumieren. Ganz analog verhält es sich mit den Target2-Salden. Der zwischen den Target2-Salden und der Leistungsbilanz konstruierte Zusammenhang ist also rein hypothetischer Natur.

Der rasante Anstieg des deutschen Target2-Saldos liegt am Status Deutschlands als sicherem Hafen im Euroraum. Anleger ziehen ihr Geld von griechischen, irischen, italienischen und anderen Banken ab und transferieren es nach Deutschland, Luxemburg und in weitere als sicher geltende Länder. Da Banken in den von Kapitalflucht betroffenen Ländern Kredite langfristig vergeben haben, müssen sie den Abfluss von Kundeneinlagen entweder über die Reduzierung des Kreditvolumens oder über die Gewinnung neuer Einlagen kompensieren. Weil der Kapitalmarktzugang vieler Banken in den Peripherieländern derzeit erschwert ist, ${ }^{11}$ wird die abgeflossene Liquidität durch Vergabe von Zentralbankkrediten ausgeglichen. Die Target2-Salden helfen somit, Bankenzusammenbrüche und Kreditkündigungen aufgrund von Illiquidität zu vermeiden. Der steigende Target2-Saldo in Deutschland und Luxemburg ebenso wie die hohen negativen Werte der Peripherie sind der Ausdruck von Kapitalflucht und nicht ein Indiz für einen weiterhin überschwänglichen Lebensstil.

Repräsentiert die Kapitalflucht nach Deutschland nun die neue Stärke Deutschlands? Die aktuell hohe Wettbewerbsfähigkeit Deutschlands, symbolisiert durch relativ niedrige Arbeitslosigkeit und hohe Leistungsbilanzüberschüsse, ist zu einem Teil ebenso eine Übertreibung, wie es die Wettbewerbsfähigkeit der Peripherieländer vor der Krise war. Während die Peripherie des Euroraums in einen Teufelskreis aus Kapitalflucht, Illiquidität, Insolvenz und weiterer Kapitalflucht geraten ist, lässt

10 Vgl. Obstfeld 2012.

11 Aufgrund des Misstrauens ist der Interbankenmarkt (der Handel von Liquidität zwischen Banken) im Euroraum zeitweise fast vollständig zusammengebrochen. 
sich umgekehrt für Deutschland ein Engelskreis beobachten. Deutschland profitiert von den Kapitalzuflüssen, die zu hoher Liquidität, niedrigen Zinsen und steigenden Investitionen und Konsum führen. Das resultierende Wachstum schafft noch stärkeres Vertrauen mit weiteren Kapitalzuflüssen.

Diese zwei sich selbst verstärkenden Prozesse verdeutlichen die Wichtigkeit von Vertrauen bei multiplen Gleichgewichten. Zudem zeigen sie, dass fundamentale Faktoren wie Wirtschaftsstruktur, Bildungsniveau oder natürliche Ressourcen die aktuelle Entwicklung allein nicht erklären können. Dementsprechend bleibt festzuhalten, dass es gefährlich wäre, die aktuelle Stärke Deutschlands als Zeichen der Stabilität zu interpretieren. Sie spiegelt nur die relative Schwäche der anderen Länder im Euroraum wider, die sich auch in einem für die deutsche Exportwirtschaft günstigen Wechselkurs gegenüber dem Rest der Welt niederschlägt. Wenn die Diskussion über die Eurokrise in Deutschland derzeit aus einer Position der Stärke geführt wird, darf nicht übersehen werden, dass es sich dabei um eine labile Position handelt, die sich sehr schnell wieder ändern kann.

Die Target2-Salden sind also kein Problem, welches aufgrund ihrer Dimension eine deutsche Abkehr vom Euro rechtfertigen würde, sondern im Gegenteil das Symptom eines zunehmenden Vertrauensverlustes in den Fortbestand der Europäischen Währungsunion. Viele Kommentatoren betonen immer wieder die unüberschaubaren Risiken der Target2-Salden, ignorieren dabei aber, dass solche Risiken erst bei einem Auseinanderbrechen der Währungsunion realisiert werden. Erst dann wird aus dem Target2-Verrechnungsposten in der Bilanz der Bundesbank eine Kreditposition mit Ausfallrisiko. Bei einem Austritt eines Landes aus dem Euroraum müssten ausstehende Verbindlichkeiten von den verbleibenden nationalen Notenbanken entsprechend ihres Kapitalschlüssels an der EZB getragen werden - für Deutschland als größter Volkswirtschaft im Euroraum beträgt dieser Kapitalanteil 27 Prozent. Der Nettobetrag der ausstehenden Verbindlichkeiten ist mit Vermögenswerten zu verrechnen. Der Deutschen Bundesbank würden im Zusammenhang mit Target 2 dann Verluste entstehen, wenn sich gleichzeitig auch die vom Bankensektor des ausgetretenen Landes hinterlegten Sicherheiten als nicht hinreichend werthaltig erweisen - etwa weil die als Sicherheit hinterlegten Staatsanleihen von dem betroffenen Land nicht mehr bedient werden. Wenn, wie vorher beschrieben, die Target2-Salden im Kern als Symptom eines fortschreitenden Vertrauensverlustes in den Zusammenhalt der Währungsunion zu deuten sind, ergibt sich so die paradoxe Situation, dass sowohl die Höhe des Risikos als auch dessen Eintrittswahrscheinlichkeit umso stärker steigt, je mehr in der öffentlichen Debatte in Deutschland Zweifel am Fortbestand des Euro gestreut werden.

Grundsätzlich gibt es nur zwei Alternativen, um ein weiteres Anwachsen der Target2-Salden zu verhindern. Die erste besteht im sofortigen Ausgleich der Target2Salden. Wer dies vorschlägt, der fordert nichts anderes als das Ende des Euro. Die zweite Alternative besteht darin, grundlegende Reformen voranzutreiben, die das Vertrauen in die Währungsunion und in das Wachstumspotential der Peripherieländer wiederherstellen. Die Konsequenzen beider Alternativen sind sorgfältig zu bedenken. In der Folge werden die beiden Optionen sowie deren wahrscheinliche Auswirkungen dargestellt. 


\section{Die Folgen eines Zusammenbruchs des Euroraums}

Viele Ökonomen ${ }^{12}$ propagieren im Kontext der Krise in Griechenland den Austritt aus dem Euroraum als einfachste Lösung. Dahinter steht die Vorstellung, ein solcher Austritt sei mit der Abwertung in einem Währungsverbund vergleichbar wie etwa im Europäischen Währungssystem der 1980er Jahre oder mit der Aufkündung einer Wechselkursbindung wie im Fall des argentinischen Peso an den US-Dollar Anfang dieses Jahrtausends. Diese Argumentation verkennt aber, dass der Austritt aus einem einheitlichen Währungsraum etwas grundsätzlich anderes ist als die Abwertung einer Währung gegenüber einer anderen. Häufig wird auf Argentinien verwiesen als Beispiel für die segensreichen Wirkungen einer Abkoppelung. Offensichtlich sehen selbst manche Wirtschaftshistoriker nicht den fundamentalen Unterschied zwischen der vergleichsweise relativ schmerzlosen Auflösung einer Wechselkursbindung und dem (chaotischen) Auseinanderbrechen einer Währung. Es gibt nur wenige historische Beispiele für einen solchen Prozess - wie den Zerfall des Österreich-Ungarischen Kaiserreichs oder das Beispiel Jugoslawien - und immer ging dies mit einer politischen Desintegration der betroffenen Regionen einher.

Dem Plädoyer für einen Austritt liegt eine zentrale Annahme zugrunde: Wenn Griechenland wieder die Drachme einführen würde, könnte es gegenüber dem Euro abwerten, seine Exportgüter verbilligen und damit die Wirtschaft wettbewerbsfähiger machen. Ein Austritt mag aus Sicht Griechenlands auf den ersten Blick als einfache Lösung erscheinen. Da nicht nominale Rigiditäten, sondern strukturelle Defizite den Kern des griechischen Problems darstellen, würden schmerzhafte und weitreichende politische Reformen hierdurch jedoch nicht vermieden, sondern nur aufgeschoben. Auch nach einer Abwertung stünde Griechenland genauso wie heute vor der Herausforderung, glaubwürdige Institutionen aufzubauen, insoweit es Vertrauen für langfristige Investitionen schaffen will. In- und ausländische Unternehmer werden keine Investitionen tätigen, solange hohe Unsicherheit über die institutionellen Rahmenbedingungen herrscht. Werden dagegen institutionelle Reformen mit Unterstützung und Anleitung der Partner innerhalb des Euroraums durchgeführt, ist ein glaubwürdiger Neustart wesentlich leichter umzusetzen.

Ein Austritt droht darüber hinaus für die anderen Partner der Währungsunion eine gefährliche Dynamik in Gang zu setzen. Die Option des Austritts eines einzelnen Landes der Peripherie aus dem Euroraum bei Fortbestehen des Euro in seiner heutigen Form im restlichen Euroraum ist deshalb kein glaubwürdiges Szenario, weil er fatale Folgen für die Dynamik der Finanzströme innerhalb des verbleibenden Euroraums hätte. Sobald einmal ein Land aus dem Euroraum ausgetreten ist, würden Investoren auch für Finanzanlagen in anderen Peripheriestaaten hohe Prämien für das potentielle Austrittsrisiko verlangen (egal ob für Staatsanleihen oder private Investitionen) und höhere Zinssätze fordern. Auch Privatanleger würden ihre Einlagen bei den lokalen Banken abziehen und in andere Regionen umschichten. Die steigenden Finanzierungskosten würden die Last der bestehenden Schulden verteuern.

12 Vgl. etwa Roubini 2011; Voth 2011.

Leviathan, 40. Jg., 2/2012 
Schon die Forderung nach einem Euroaustritt verteuert also die Finanzierung für alle Peripherieländer, öffnet die Tür für spekulative Kapitalbewegungen und verschlechtert über den weiteren Rückgang des Vertrauens die Wettbewerbsfähigkeit der betroffenen Länder. Bemühungen von EZB und Regierungen für eine Verbesserung der wirtschaftlichen Situation werden so torpediert. Dies ist deshalb so brisant, weil sich die Risiken endogen entwickeln - sie hängen entscheidend davon ab, welche Politik betrieben wird. Die Forderung nach einem Austritt ist ein Spiel mit dem Feuer. Damit würde die Kreditklemme in den Peripherieländern noch weiter verschärft und die Kapitalflucht beschleunigt. Eine Verpflichtung zum raschen Ausgleich der Target2-Salden würde nichts anderes bedeuten als das abrupte Ende der Währungsunion. Die dann unvermeidliche Einführung massiver Kapitalverkehrskontrollen wäre für Anleger in der ganzen Welt das deutliche Signal, dass Europa nicht in der Lage ist, sein eigenes Haus in Ordnung zu bringen. Wer Zweifel am Fortbestand des Euro sät, potenziert gerade die Risiken, die er beklagt.

Manche Ökonomen betonen, ein Austritt könnte ja auch nur temporär erfolgen, bis das betreffende Land seine Wettbewerbsfähigkeit wiederhergestellt und seine Staatsfinanzen saniert hätte. Bei dieser Sichtweise wird jedoch verkannt, dass die Kosten eines temporären Euroaustritts eines Eurostaates keineswegs geringer ausfallen dürften als die eines dauerhaften Austritts. Ob temporär oder nicht: Der Austritt eines Landes wäre ein Präzedenzfall, welcher das Vertrauen in den Währungsraum dauerhaft schwer beschädigen würde. Ein gemeinsamer Währungsraum ist eben keine Mitgliedschaft wie die in einer beliebigen Organisation. Damit ein einheitlicher Währungsraum jene Vorteile bringen kann, für deren Realisierung er einst gegründet wurde, ist unabdingbare Voraussetzung, dass er auf Dauer angelegt ist. Kann man auf den Fortbestand des Währungsraums nicht vertrauen, werden zwangsläufig für grenzüberschreitende Transaktionen Risikoprämien erhoben, die kostspielige Absicherungsgeschäfte für Unternehmen erfordern und eine endogene Instabilität schaffen. Schon kleinere Krisen einzelner Mitgliedstaaten können dann ausreichen, Spekulationen um deren Austritt oder Ausschluss aus der Währungsunion zu befeuern. Solange ein reibungsloser Zahlungsverkehr garantiert wird, induziert dies gewaltige Kapitalbewegungen, welche die aus dem Vertrauensverlust erwachsenen Krisen dramatisch intensivieren und unvermeidlich zur Einführung massiver Kapitalverkehrskontrollen zwingen würde. Kurz: Ein Euroaustritt, ob temporär oder nicht, würde explosive Dynamiken hervorrufen, denen der Euroraum (zumindest ohne dramatische Reformen) wohl dauerhaft kaum gewachsen wäre. Ein Währungsraum »auf Abruf « kann nicht funktionieren. Treffend hat dies der langjährige Vorsitzende des Sachverständigenrats Olaf Sievert ausgedrückt: »Es gibt Projekte, die muss man letztlich ohne Absicherung wagen - oder lassen. Die Ehe alter Art etwa. Man muss sie sich selbst zutrauen, nicht den anderen. Die Europäische Währungsunion ist von dieser Art «.13 


\section{Die Alternative: Institutionelle Weiterentwicklung Europas}

Die Alternative zu diesem Szenario kann jedoch nicht in einem »weiter so wie bisher « bestehen. Denn die Krise hat auf dramatische Art und Weise die Mängel und Unvollkommenheiten der Europäischen Währungsunion aufgedeckt. Schon vor Einführung des Euro haben zahlreiche Ökonomen immer wieder auf das Grundproblem hingewiesen: das Fehlen einer zentralen Fiskalinstanz, die - mit Besteuerungs- und Ausgabekompetenzen versehen - die Wirkung asymmetrischer Schocks ausgleichen und gleichzeitig der Integration der europäischen Finanzmärkte ein angemessenes politisches Gegengewicht entgegenstellen könnte. Eine solche Fiskalunion war bei Einführung des Euro politisch noch nicht durchsetzbar. Anstelle dessen sollte der von Deutschland durchgesetzte Vertrag von Maastricht als eine Art »minimal fiscal Europe « durch die Regelbindung der nationalen Haushaltspolitik zumindest ein Minimum an fiskalischer Konvergenz und Koordination gewährleisten. Dieser Versuch muss mit der derzeitigen Eurokrise als vollständig gescheitert angesehen werden. Dies liegt nicht, wie von einigen Ökonomen immer wieder argumentiert wird, daran, dass der Pakt seit dessen Reform im Stabilitäts- und Wachstumspakt 2003 zum zahnlosen Tiger wurde. Dies verdeutlicht unsere Diskussion der Staatsverschuldungskrise im Euroraum: Gerade ehemalige Musterländer des Stabilitäts- und Wachstumspakts wie Spanien und Irland stehen heute vor großen Problemen, während Länder wie Deutschland oder Frankreich, die den Pakt wiederholt gebrochen haben, zurzeit vergleichsweise gut dastehen.

Nein, das Scheitern des Pakts geht vielmehr darauf zurück, dass dessen Kriterien nicht ausreichten, um das für eine Währungsunion notwendige Niveau fiskalischer Konvergenz sicherzustellen. So versteift sich der Stabilitäts- und Wachstumspakt ausschließlich auf die Staatsverschuldung, ohne gemeinverbindliche Regeln für andere wichtige Bereiche wie die Steuerpolitik, Wettbewerbs- und Investitionspolitik oder die Ausgestaltung sozialer Sicherungssysteme vorzusehen. Fehlentwicklungen im Bereich der Verschuldung privater Haushalte konnte so ebenso wenig gegengesteuert werden wie der Auseinanderentwicklung der Wettbewerbsfähigkeit der Eurostaaten. Erst durch diesen blinden Fleck der europäischen Institutionen war es möglich, dass sich (wie eingangs aufgezeigt) die Wettbewerbsfähigkeit innerhalb des Euroraums so dramatisch auseinanderentwickelte. Während man den Finanzmärkten bei der Staatsverschuldung misstraute und durch eine Regelbindung potentiellen Fehlentwicklungen vorzubeugen hoffte, setzte man in diesen Bereichen voll auf die Effizienz der Kapitalmärkte - mit bekanntem Ergebnis.

Die Krise wurde also nicht durch ein »zu viel Europa " verursacht, sondern im Gegenteil durch die Unfähigkeit der Mitgliedstaaten, der europäischen Zentralbank eine vergleichbare Fiskalinstanz zur Seite zu stellen und der währungspolitischen Union die politische Union folgen zu lassen. Dies zeigt klar die Aufgabe, der sich die Politiker heute stellen müssen, um den dauerhaften Fortbestand des Euroraums zu sichern. Der europäischen Währungsunion muss mit einer Fiskalunion endlich das zweite Standbein hinzugefügt werden, das sie in die Lage versetzt, auch in turbulenten und krisenhaften Zeiten aufrecht stehen zu können. 
Eine europäische Fiskalunion wäre auch das geeignete Mittel, um der Target2Problematik zu begegnen. Die Existenz einer handlungsfähigen europäischen Fiskalinstanz würde die Europäische Zentralbank von der Bürde entlasten, bei der Unterstützung wackelnder Finanzinstitute auch Solvenzrisiken auf ihre Bilanz nehmen zu müssen. Die EZB würde somit in substanzieller Weise entlastet und könnte sich voll auf ihre Aufgabe der Geldwertstabilität konzentrieren, ohne dramatische Verwerfungen auf den Finanzmärkten befürchten zu müssen. In dem Maße, wie durch die Schaffung einer Fiskalunion das Vertrauen in die Wettbewerbsfähigkeit der Krisenstaaten und den Fortbestand des Euroraums gestärkt werden würde, würde auch die Kapitalflucht aus den Krisenstaaten enden; damit könnte eine Normalisierung der Target2-Salden eingeleitet werden.

Die Unterzeichnung des Fiskalpakts als Weiterentwicklung des Stabilitäts- und Wachstumspakts stellt einen ermutigenden ersten Schritt in diese Richtung dar. Dieser reicht jedoch bei weitem nicht aus. Zum einen muss das Feld der abstimmungsbedürftigen Gebiete über das der nationalen Defizitpolitik hinaus ausgeweitet werden, zum anderen sollte auch ein Transfer nationaler Zuständigkeiten auf die supranationale europäische Ebene stattfinden. Eine europäische Fiskalunion kann durchaus unterschiedliche Formen annehmen. Wie von namhaften Ökonomen ${ }^{14}$ aufgezeigt, muss der Transfer nationaler Hoheitsrechte nicht dramatisch ausfallen, um eine effektive europäische Fiskalunion herbeizuführen. Auf alle Fälle wären die direkten ökonomischen Kosten (von den politischen Kosten ganz zu schweigen), die dies für Deutschland implizieren würde, um ein Vielfaches geringer als bei der Alternative des Auseinanderbrechens des Euroraums, denn ein schlichtes »weiter so wie bisher « ist angesichts der anstehenden Refinanzierungsvolumina keine Option.

Das heutige Europa ist das Resultat einer Aufeinanderfolge von Krisen. Diese haben Europa seine heutige Gestalt gegeben. Auch die Europäische Währungsunion in ihrer heutigen Form ist das Resultat von Krisen - von der Krise des Europäischen Wechselkursverbunds Ende der 1970er bis zur Krise des Europäischen Währungssystems Anfang der 1990er Jahre. Im Einklang mit guter europäischer Tradition geht es darum, als Reaktion auf die derzeitige Krise Europa weiterzuentwickeln und zu stärken, anstatt Zuflucht zu nationalen Lösungen zu suchen. Gerade in Krisenzeiten ist die Neigung, sich auf die Ebene des Nationalstaats zu flüchten, besonders ausgeprägt. Angesichts der globalisierten Finanzmärkte lässt sich ein Handlungsspielraum der Politik aber nur auf europäischer Ebene wiedergewinnen.

\section{Literatur}

Bindseil, Ulrich; Koenig, Philipp Johann 2011. The economics of TARGET2 balances. SFB 649 Discussion Paper 2011-035. Berlin: Humboldt-Universität zu Berlin.

Blankart, Charles B. 2012. »Deutschland in der Target-Falle«, in Ökonomenstimme, 23. März 2012. http://www.oekonomenstimme.org/artikel/2012/03/deutschland-in-der-target-fallegriechenland/ (Zugriff vom 05.04.2012).

14 Vgl. Marzinotto et al. 2011; Bordo et al. 2011. 
Bordo, Michael; Markiewicz, Agnieszka; Jonung, Lars 2011. A fiscal union for the euro: some lessons from history. NBER Working Paper No. 17380. Cambridge: National Bureau of Economic Research.

Bornhorst Fabian; Mody, Ashoka 2012. »TARGET imbalances: financing the capital-account reversal in Europe ", in VOX - research-based policy analysis and commentary from leading economists, 7. März 2012. http://voxeu.org/index.php?q=node/7700 (Zugriff vom 05.04.2012).

Bruno, Valentina; Shin, Hyun 2012. Capital flows, cross-border banking and global liquidity. Working Paper. Princeton: Princeton University.

De Grauwe, Paul 2011. The governance of a fragile Eurozone. CEPS Working Document No. 346. Brüssel: Centre for European Policy Studies.

Eichengreen, Barry 2012. »Implications of the Euro's crisis for international monetary reform «, in Journal of Economic Policy Modeling (forthcoming).

Marzinotto, Benedicta; Sapir, André; Wolff, Guntram B. 2011. What kind offiscal union? Bruegel Policy Brief 2011/06. http://www.bruegel.org/publications/publication-detail/publication/ 646-what-kind-of-fiscal-union/ (Zugriff vom 16.04.2012).

Obstfeld, Maurice 2012. Does the current account still matter? NBER Working Paper No. 17877. Cambridge: National Bureau of Economic Research.

Roubini, Nouriel 2011. "Greece should default and abandon the euro", in Financial Times, 19. September 2011.

Sargent, Thomas J. 2011. United States then, Europe now. Nobel Prize Lecture in Economics, Stockholm, 8. Dezember 2011.

Sievert, Olaf 2012. "Offener Brief an Hans-Werner Sinn ", in Wirtschaftliche Freiheit, 3. März 2012. http://wirtschaftlichefreiheit.de/wordpress/?p=8787 (Zugriff vom 05.04.2012).

Sinn, Hans-Werner; Wollmershaeuser, Timo 2011. Target-Kredite, Leistungsbilanzsalden und Kapitalverkehr: Der Rettungsschirm der EZB. ifo Working Paper No. 105, Juni 2011. München: ifo Institut.

Voth, Hans Joachim 2011. "Der Euro wird scheitern«, in Wirtschaftswoche, 19. November 2011. 
Zusammenfassung: Das Kernproblem der aktuellen Eurokrise liegt in der Kapitalflucht aus den Peripheriestaaten, welche zu einem inferioren Gleichgewicht mit starkem Wirtschaftseinbruch führt. Forderungen nach einem Austritt aus dem Euroraum beschleunigen diese Kapitalflucht und potenzieren so die Risiken. Die Lösung des Problems liegt in einer institutionellen Weiterentwicklung Europas mit einer zentralen Fiskalinstanz, die der Integration der Finanzmärkte ein angemessenes politisches Gegengewicht entgegenstellt.

Stichworte: Eurokrise, Europäische Zentralbank, Fiskalunion, Kapitalflucht, Kreditklemme

\title{
The discussion about the Euro crisis and its consequences
}

Summary: The current Euro crisis is due to a massive capital flight from peripheral countries that has induced a credit crunch and degraded equilibrium. Calls to withdraw from the Euro area only accelerate this capital flight and thereby amplify the risks. The adequate response consists in strengthening the supranational level and in complementing European financial integration with an effective European fiscal union.

Keywords: Euro crisis, European Central Bank, fiscal union, capital flight, credit crunch

\author{
Autoren \\ Prof. Dr. Gerhard Illing \\ Seminar für Makroökonomie \\ Ludwig-Maximilians-Universität München \\ Ludwigstraße $28 \mathrm{Rg}$. \\ 80539 München \\ E-Mail: Illing@lmu.de \\ Sebastian Jauch \\ Seminar für Makroökonomie \\ Ludwig-Maximilians-Universität München \\ Ludwigstraße $28 \mathrm{Rg}$. \\ 80539 München \\ Sebastian.Jauch@lrz.uni-muenchen.de \\ Michael Zabel \\ Seminar für Makroökonomie \\ Ludwig-Maximilians-Universität München \\ Ludwigstraße $28 \mathrm{Rg}$. \\ 80539 München \\ Michael.Zabel@1rz.uni-muenchen.de
}

